La Revista Panamericana de Salud Pública/Pan American Journal of Public Health se complace en publicar cartas de los lectores dirigidas a estimular el diálogo sobre los diversos aspectos de la salud pública en las Américas, así como a esclarecer, discutir o comentar de manera constructiva las ideas expuestas en la revista. Las cartas deben estar firmadas por el autor y especificar su afiliación profesional y dirección postal. Cuando se trate de comentarios sobre un artículo que requieran contestación del autor, se procurará conseguir esa respuesta con el fin de publicar ambas cartas. La Redacción se reserva el derecho de editar las cartas recibidas y resumirlas para mayor claridad.

The Revista Panamericana de Salud Pública/Pan American Journal of Public Health publishes letters from readers for the purpose of stimulating dialogue on various aspects of public health in the Americas and of constructively clarifying, discussing, and critiquing the ideas expressed throughout its pages. Letters should be signed by the author and include his or her professional affiliation and mailing address. If a commentary on a given article requires a reply from the author, an effort will be made to obtain the reply and to publish both letters. The editorial team reserves the right to edit all letters received and to condense them so as to improve their clarity.

\section{REFLEXÕES SOBRE O “SEDENTARISMO” NA PESQUISA SOBRE FATORES DE RISCO PARA INFARTO AGUDO DO MIOCÁRDIO}

No final de 2007, a Revista Panamericana de Salud Pública trouxe a síntese (1) de um recémpublicado estudo (2) sobre os fatores de risco para o infarto agudo do miocárdio (IAM) na América Latina. Considerando a quantidade de mortes relacionadas às enfermidades cardiovasculares, esse estudo de caso-controle objetivou esmiuçar a força dos fatores de risco em indivíduos na América Latina que haviam desenvolvido IAM pela primeira vez. Os autores concluíram que os mais importantes fatores de risco haviam sido a obesidade abdominal, o tabagismo, a dislipidemia, a hipertensão, o consumo inadequado de frutas e outros vegetais e a falta de regularidade ou a não prática de exercícios físicos. Ainda segundo os autores, a prática regular de exercícios físicos oferece um relevante efeito protetor, principalmente se combinada com a prevenção de outros fatores de risco (2).

Em que pese o fato de a prática insuficiente de exercícios físicos não ter sido o mais importante fator de risco, é indispensável ponderar que há muito esse hábito vem sendo considerado essencial pelos especialistas (3). Contudo, há pouca reflexão sobre algumas questões relacionadas ao tema.

Um primeiro aspecto merecedor de aprofundamento diz respeito à dificuldade decisiva de se determinar a ocorrência de sedentarismo, uma vez que os critérios de análise são grosseiramente dessemelhantes e inócuos. É possível encontrar quem defina o sedentarismo simplesmente como a falta de prática de exercícios físicos; outros se detêm nas atividades físicas realizadas nas horas de lazer; há quem entenda que o sedentarismo se relaciona à quantidade arbi- trária de atividades físicas do cotidiano $(4,5)$. Nesse sentido, merece destaque o fato de os pesquisadores estarem se valendo de critérios díspares e incertos para tirar conclusões sobre incidências e associações. Lanas et al. (2), na investigação supracitada, consideraram como fisicamente ativos os indivíduos que se envolveram regularmente com algum exercício moderado ou extenuante por 4 ou mais horas por semana. Contudo, é razoável perguntar se alguns sujeitos não acabaram deixando de ser classificados como "ativos" mesmo realizando esforço físico exagerado em suas tarefas cotidianas, fato, inclusive, bastante provável na amostra investigada.

Outro ponto digno de reflexão pode ser traduzido no questionamento elaborado por MagallanesMira (6): é o exercício físico que gera a saúde ou é a saúde que conduz ao exercício? A associação entre o sedentarismo, revelado por algum critério duvidoso, e uma provável enfermidade tem sido tratada como uma relação de causalidade. Entretanto, pouco temos nos debruçado sobre a possibilidade de serem os sujeitos que buscam a prática de exercícios físicos os mesmos que apresentam menos agravos à saúde.

Algumas investigações, porém, podem ajudar nesta reflexão. Myers et al. (7), a partir de 6213 testes ergométricos aplicados em homens, observaram que a capacidade para realizar o exercício físico foi o mais importante fator preditivo de mortalidade para doenças cardiovasculares. Esses autores defenderam que tal capacidade foi maior entre os sujeitos que não haviam falecido durante o período de seguimento, considerando tanto aqueles julgados "assintomáticos" quanto os portadores de doenças cardiovasculares, independentemente da prática de exercícios. A despeito de a aptidão física freqüentemente estar relacionada ao treinamento físico sistemático, há 
uma razoável suspeita de que uma condição genética favorável possa resultar em níveis mais elevados de aptidão física (8) e, nesse caso, seria possível que essa mesma condição genética pudesse favorecer uma melhor "saúde" cardiovascular.

Essa idéia foi analisada por Wisløff et al. (9) e, de certo modo, põe em xeque qualquer tentativa de se estabelecer causalidade entre o sedentarismo e a ocorrência de doenças. Wisløff et al. (9) selecionaram artificialmente, em 11 gerações, uma população de ratos com elevada capacidade aeróbia e outra com baixa capacidade aeróbia. Os autores verificaram que os animais com baixa capacidade aeróbia, além da diminuição dessa capacidade em si, apresentavam maior predisposição às doenças cardiovasculares e metabólicas, tais como pressão arterial elevada, disfunção endotelial, liberação reduzida de insulina, maior adiposidade visceral e níveis mais elevados de triglicerídeos, mesmo quando submetidos a treinamento físico.

Além disso, como ressalta Lupton (10), o discurso sobre o risco pode ser analisado sob duas grandes perspectivas. A primeira compreende as ameaças externas à saúde da população — tais como a poluição do ambiente, os resíduos nucleares ou químicos, os desastres naturais -, sobre as quais os sujeitos, isoladamente, têm pouca ou nenhuma ingerência. Por outro lado, uma segunda configuração foca o risco como conseqüência das escolhas individuais do "estilo de vida" e enfatiza as responsabilidades e autocontrole para gerenciar os perigos à saúde. Nesse sentido, nas sociedades contemporâneas, em nome da saúde, tem-se esperado dos indivíduos que tenham responsabilidade para cuidar de seus corpos e, entre outras atitudes, temse cobrado o engajamento em programas de exercícios físicos. A autora lembra, porém, que os discursos e as práticas de caráter biomédico presentes na saúde pública criam objetos e campos de interesse (doenças, sofrimentos, pacientes, medicamentos, técnicas de reabilitação ou restauração, etc.) que ajudam a constituir e regular os fenômenos como "normais", de "risco" e "saudáveis"; dessa forma, como um sistema moral, essas práticas esboçam distinções entre os "bons" ou "maus" pacientes. Nesse sentido, talvez seja imperativo questionar se as escolhas são possíveis ou desejáveis; ou, ainda, se as atitudes são manifestações de reação à precariedade da vida, isto é, modos de se defender frente à intensificação dos desgastes físicos do diaa-dia, ou mesmo de buscar o prazer. Ademais, o termo sedentarismo, quase sempre, assume simbolicamente um tom pejorativo e discriminatório. A própria noção de risco, por sua vez, pode ser construída às avessas. Não raro, na literatura científica, nos deparamos com textos que indicam a possibilidade de o exercício representar risco à saúde (11-13). Dessa forma, ao mesmo tempo que mais estudos deveriam ser conduzidos, mais acirradas de- veriam ser as reflexões acerca dos sentimentos de culpa e angústia ilimitados e espargidos através dos riscos construídos pelo estatuto epidemiológico.

Alexandre Palma

Universidade Federal do Rio de Janeiro Escola de Educação Física e Desportos Avenida Carlos Chagas Filho, 540

Edifício da Escola de Educação Física e Desportos Cidade Universitária, Ilha do Fundão CEP 21941-599, Rio de Janeiro, RJ, Brasil

Fone: +55-21-2562.6804

E-mail: palma_alexandre@yahoo.com.br

Monique Assis

Sociedade Universitária Augusto Motta Curso de Educação Física

Brasil

\section{Referências}

1. Factores de riesgo de infarto agudo del miocardio en América Latina. Rev Panam Salud Publica. 2007;22(6):402.

2. Lanas F, Avezum A, Bautista LE, Diaz R, Luna M, Islam S, et al. Risk factors for acute myocardial infarction in Latin America. Circulation. 2007;115(9):1067-74.

3. US Department of Health and Human Services. Physical activity and health: a report of the surgeon general. Atlanta, GA: US Department of Health and Human Services, Centers for Disease Control and Prevention, National Center for Chronic Disease Prevention and Health Promotion; 1996.

4. Varo JJ, Martínez-González MA, Irala-Estévez J, Kearney J, Gibney M, Martinez A. Distribution and determinants of sedentary lifestyles in the European Union. Int J Epidemiol. 2003;32(1):138-46.

5. Monteiro CA, Conde WL, Matsudo SM, Matsudo VR, Bonseñor IM, Lotufo PA. A descriptive epidemiology of leisuretime physical activity in Brazil, 1996-1997. Rev Panam Salud Publica. 2003;14(4):246-54.

6. Magallanes-Mira C. Exercício físico e saúde: da crítica prudente. Em: Bagrichevsky M, Palma A, Estevão A, orgs. A saúde em debate na educação física. Blumenau: Edibes; 2003. Pp.169-91.

7. Myers J, Prakash M, Froelicher V, Do D, Partington S, Atwood E. Exercise capacity and mortality among men referred for exercise testing. N Engl J Med. 2002;46(11):793-801.

8. Wolfarth B, Bray MS, Hagberg JM, Perusse L, Rauramaa R, Rivera MA, et al. The human gene map for performance and health-related fitness phenotypes: the 2004 update. Med Sci Sports Exerc. 2005;37(6):881-903.

9. Wisløff U, Najjar SM, Ellingsen Ø, Haram PM, Swoap S, Al-Share $\mathrm{Q}$, et al. Cardiovascular risk factors emerge after artificial selection for low aerobic capacity. Science. 2005;307(5708):418-20.

10. Lupton D. The imperative of health: public health and the regulated body. London: Sage; 1997.

11. Buist I, Bredeweg SW, Lemmink KA, Pepping GJ, Zwerver J, van Mechelen W, et al. The GRONORUN study: is a graded training program for novice runners effective in preventing running related injuries? Design of a randomized controlled trial. BMC Musculoskelet Disord. 2007;8:24.

12. Fransson E, Faire U, Ahlbom A, Reuterwall C, Hallqvist J, Alfredsson L. The effect of leisure-time physical activity on the risk of acute myocardial infarction depending on body mass index: a population-based case-control study. BMC Public Health. 2006;6:296.

13. American College of Sports Medicine; American Heart Association. Exercise and acute cardiovascular events: placing the risks into perspective. Med Sci Sports Exerc. 2007;39(5): 886-97. 